6 Barbir M, Banner N, Thompson GR, Khagan A, Mitchell A, Yacoub M. Relationship of immunosuppression and serum lipids to the development of coronary arterial disease in the transplanted heart. Int $f$ Cardiol 1991;32:51-6.

7 Eich D, Thompson JA, Ko D, et al. Hypercholesterolaemia in long term survivors of heart transplantation: An early marker of accelerated coronary artery disease. F Heart Lung Transplant 1991,10.45-9.

McDonald K, Rector TS, Braunlin EA, Kub SH, Olivari MT. Association of coronary artery disease in cardiac transplant recipients with cytomegalovirus infection. $A m f$ Cardiol 1989;64:359-62.

9 Carpier GL, Jones PH, Suki WN, et al. Rhabdomyolysis and renal injury with lovastatin use. $\mathscr{f} A M A$ 1988;260:239-41.

A characteristic continuous wave Doppler signal in cor triatriatum?

SIR-Alwi et al (British Heart fournal 1992;68:6-8) state that "Previous reports in English merely mentioned high velocity diastolic signals when the pulsed Doppler sample volume was placed beneath the restrictive orifice."

This statement is inaccurate. We gave an accurate description of the haemodynamics in this rare anomaly including colour Doppler plates of the exact flow directions in early and late systole and diastole in a case that we reported in 1990 .

JORAM GLASER Paediatric Cardiology Uni, Shaare Zedek Medical Centre,
ferusalem, Israel

1 Glaser J, Goren A, Ilan M, Vidne B. Cor triatriatum sinistrum: Diagnosis by Doppler
echocardiography. Cardiology 1990;77: $472-6$

\section{The future of paediatric cardiology in} the United Kingdom

SIR,-As chairman of the working party that produced the report on paediatric cardiology (British Heart fournal 1992;68:630-3) may I make a small but important amendment. This report has been a long time in gestation and I am happy to say that during the last "trimester" paediatric cardiology has been born again in Wales at the University Hospital of Wales in Cardiff The information regarding paediatric cardiology in Cardiff contained in the report is thus out of date.

STEWART HUNTER

Department of Paediatric Cardiology

Freeman Hospital, High Heaton Freeman Hospital, High Heaton,
Nercastle upon Tyme NE7 7DN
Propionibacterium acnes causing perivalve abscess

SIR,-Dr Horner and colleagues described their experience with a rare case of Propionibacterium acnes endocarditis associated with an aortic root abscess. ${ }^{1}$ We have recently seen a similar case of prosthetic mitral valve endocarditis with operative evidence of healed perivalvar abscesses and were impressed by certain features common to both cases which are worthy of comment in this rare infection.

A 57 year old woman was admitted to hospital with severe mitral regurgitation and suspected infective endocarditis in August 1992. In 1981 she had undergone mitral valve replacement with a Starr-Edwards prosthesis for rheumatic heart disease. In 1991, eighteen months before admission she had developed progressive dyspnoea and a cough accompanied by a raised erythrocyte sedimentation rate and a mitral regurgitant murmur. One month before admission she also experienced weight loss. Five sets of blood cultures produced a growth of Propionibacterium acnes. The patient was clinically stable, but before valve replacement was undertaken satisfactory control of sepsis was desired. Valve replacement was deemed necessary because of the degree of regurgitation observed from this prosthesis. The organism isolated was susceptible to penicillin, ampicillin, vancomycin, and erythromycin and was less susceptible to rifampicin and ciprofloxacin. Minimum inhibitory concentrations were $0.5 \mathrm{mg} / \mathrm{l}, 0.12 \mathrm{mg} / \mathrm{l}, 2.0 \mathrm{mg} / \mathrm{l}, 1.0 \mathrm{mg} / \mathrm{l}, 2.0$ $\mathrm{mg} / \mathrm{l}$, and $2.0 \mathrm{mg} / \mathrm{l}$ respectively. Thus treatment with benzyl penicillin and gentamicin was started with the aim of later elective valve replacement. The fever resolved but despite satisfactory serum gentamicin concentrations, symptoms of dizziness developed after three weeks' combination therapy. Benzyl penicillin alone (2 MU every four hours) was given until elective replacement of the mitral valve prosthesis was performed a week later. At operation, there were multiple tracts around the original prosthesis and, when it was removed, evidence of healing periannular abscesses with some vegetations. Cultures of the vegetations and prosthesis were sterile. The patient continued to receive benzyl penicillin 2 MU every four hours with the addition of probenecid $500 \mathrm{mg}$ twice daily during convalescence on the ward. Peak and trough serum bactericidal titres were $1 / 8$ and $<1 / 2$ respectively. Also, several blood cultures obtained when penicillin concentrations were expected to be at their lowest (that is, just before the penicillin doses) proved to be sterile. However, after three weeks, a classic rash of penicillin allergy developed which necessitated a change in antibiotic therapy to vancomycin. This was continued for a further three weeks with regular monitoring for therapeutic serum concentrations-giving a total of six weeks postoperative intravenous antibiotic therapy. The patient remains well to date.

This case illustrates the typical nature of endocarditis caused by a low grade pathogen - with a relatively long history and minimal signs of infection. In a study of antibiotic prophylaxis, 22 of 60 patients receiving conventional flucloxacillin/aminoglycoside prophylaxis for cardiac surgery had propionibacteria retrieved from the extracorporeal blood reservoir at completion, ${ }^{2}$ so contamination is potentially common but infection is rare. Could this infection have been present for 10 years before presentation? The case supports the opinion that perivalve abscess, a more common complication of prosthetic than native valve endocarditis, is not necessarily associated with more virulent microorganisms. Therefore, if the clinical response to optimum antimicrobial therapy is poor or othe signs of uncontrolled sepsis are observed, the diagnosis of abscess formation should be suspected even in endocarditis caused by a low grade pathogen. It is noteworthy that healing of the perivalvar abscesses was progressing with four weeks' treatment with intravenous antibiotics in our patient. There are no guidelines on the amount and length of antibiotic therapy that are necessary after removal of an infected prosthetic valve but we, like Horner et al, continued this for six weeks. Fear of recurrent infection leads to antibiotic therapy being prolonged. This has important consequences in terms of side effects, toxicity, and cost. Only experience can guide management. MICHAEL J MARTIN T TREASURE

Public Health Laboratory and Department of Cardiothoracic Surgery, St George's Hospital, London, SW17 OQT

1 Horner SM, Sturridge MF, Swanton RH. Propionibacterium acnes causing an aortic root abscess. Br Heart $\mathcal{F}$ 1992;68:218-20.

2 Wilson APR, Felmingham D, Gruneberg RN, Treasure T, Sturridge MF. Contamination Treasure T, Sturridge MF. Contamination of blood during cardiopulmonary bypass: Infect $1988 ; 12: 35-42$. 